\title{
Clinical management of conduction abnormalities following transcatheter aortic valve replacement: prospective evaluation of a standardized management pathway
}

\author{
Alice Haouzi ${ }^{1,2} \cdot$ Mark Tuttle $^{2} \cdot$ Allon Eyal $^{2} \cdot$ Kunal Tandon $^{2} \cdot$ Patricia Tung $^{1,2} \cdot$ Peter J. Zimetbaum $\cdot$ Daniel B. Kramer ${ }^{1,2}$
}

Received: 6 December 2021 / Accepted: 8 February 2022 / Published online: 25 February 2022

(c) The Author(s), under exclusive licence to Springer Science+Business Media, LLC, part of Springer Nature 2022

\begin{abstract}
Purpose Limited evidence guides management of conduction abnormalities following TAVR. Standardized clinical pathways may reduce variability in care while minimizing bradyarrhythmic morbidity, length of stay (LOS), and pacemaker (PPM) implantation rates.

Methods A multidisciplinary consensus pathway to standardize post-TAVR management was developed. We evaluated (1) pathway adherence; (2) LOS; (3) PPM implantation rates; (4) 1-month survival, and (5) late heart block. Exploratory analyses evaluated factors associated with PPM implantation.

Results A total of 181 consecutive patients without prior PPM who underwent TAVR between February 2020 and February 2021 (mean age $77.9 \pm 9.1,38 \%$ women) were included. Average LOS was 3.0 days ( \pm 2.7 ), and no deaths related to syncope/ bradyarrhythmia were reported by 1 month. Overall, $93 \%$ of the 181 patients were managed by pathway; deviations were due to failure of discharge with a heart monitor when it was clinically indicated for either pre-existing RBBB or new PR prolongation/new LBBB. PPM implantation occurred in 19 patients by discharge, and 21 by 1-month (13\%). In our exploratory analysis, pre-existing RBBB, transient peri-procedural heart block, and LOTUS valves were associated with pacemaker implantation: $O R(C I)$ of 8.16 (3.06-21.78), 6.83 (1.94-24.03), and 8.32 (1.11-62.49), respectively.

Conclusions This report illustrates that a standardized protocol for the management of conduction abnormalities after TAVR can be implemented with high compliance, safe management of conduction disturbance, and relatively short LOS with discharge supported by ambulatory monitoring.
\end{abstract}

Keywords Transcatheter aortic valve replacement $\cdot$ Pacemakers $\cdot$ Heart block

\section{Introduction}

Transcatheter aortic valve replacement (TAVR) was first introduced as an alternative for patients with severe aortic stenosis at high or prohibitive surgical risk, but includes nontrivial rates of permanent pacemaker implantation [1]. Over the past decade, TAVR volume has increased fivefold, with over 72,000 cases performed in 2019 [2]. This

Daniel B. Kramer

dkramer@bidmc.harvard.edu

1 Richard A. and Susan F. Smith Center for Outcomes Research in Cardiology, Beth Israel Deaconess Medical Center, 375 Longwood Ave, 4th floor, Boston, MA 02215, USA

2 Harvard Medical School, Boston, MA, USA significant rise in TAVR aligns with additional clinical trials illustrating the safety and effectiveness of the procedure in broader, lower-risk populations [3, 4]. Despite growing procedural experience and technological innovation in valve design, rates of pacemaker implantation persist in the $6.5-17 \%$ range [2-5].

Pacemaker implantation varies across studies in part because of population heterogeneity, differences in device design, and variability in clinical decision-making for patients with new conduction abnormalities. For example, while selected patient and procedural factors appear associated with post-TAVR heart block [6], no established algorithm accurately predicts progression of conduction disease or its time course. Recent expert consensus guidelines suggest maintaining transvenous temporary pacing for at least $24 \mathrm{~h}$ in higher risk patients [6], and advocate for prolonged hospital monitoring of at least 2 days after TAVR in those 
with new or worsened conduction disturbances. However, streamlined identification of high-risk patients, as well as standardization of monitoring practices, is critical to control inpatient length of stay and costs associated with the procedure.

To approach these knowledge gaps and standardize care at our institution, we developed and implemented a clinical protocol informed by cardiac electrophysiology, structural heart disease, and cardiac surgery. This report describes our multidisciplinary approach and the first 12 months of prospective, consecutive evaluation aimed at defining adherence to the protocol, observed permanent pacemaker implantation rates, and safety.

\section{Methods}

\subsection{Data source and patient population}

This study was performed at Beth Israel Deaconess Medical Center (BIDMC), an academic referral center in Boston, MA, with ethics approval provide by its institutional review board. All consecutive patients who underwent TAVR at BIDMC between 02/01/2020 and 02/28/2021 were evaluated. Patients with permanent pacemakers prior to TAVR were excluded. Clinical, electrocardiographic, and laboratory data were extracted from the online medical record.

\subsection{Management protocol}

A clinical protocol for managing post-TAVR patients at our institution was developed as a collaboration between cardiac electrophysiology, structural heart disease, and cardiac surgery. Figure 1 summarizes the clinical protocol for managing patients who underwent TAVR in accordance with pre-, peri-, and post-TAVR conduction abnormalities. All patients had temporary balloon-tipped pacing wires placed via the femoral vein for TAVR deployment. Patients with persistent complete atrioventricular (AV) block, defined as block that persisted beyond the end of the TAVR procedure, were referred for immediate pacemaker implantation, either before the patient left the hybrid operating suite or immediately after. Temporary pacemaker was placed if a permanent one could not be placed immediately following TAVR (e.g., if cardiac electrophysiology was not immediately available). Patients with transient complete AV block, defined as complete AV block that resolved prior to the end of the case, would instead undergo monitoring in the cardiac critical care unit (CCU). Patients with recurrent complete AV block were then referred for permanent pacemaker implantation.

Those with no evidence of heart block but pre-existing right bundle branch block (RBBB), new left bundle branch block (LBBB), or new PR or QRS prolongation were monitored on telemetry and discharged with a 14-day Ziopatch mobile telemetry cardiac monitor. In addition, patients with new wide LBBB $>160$ ms were offered a diagnostic EP study with direct evaluation of the conduction system. Patients with PR prolongation but not widened LBBB did not undergo EPS. In these cases, by protocol patients with a prolonged $\mathrm{HV}$ interval $>65 \mathrm{~ms}$ were referred for pacemaker implantation.

\subsection{Variables}

We collected data according to patients' demographic and clinical characteristics, procedural details, and diagnostic tests. Demographic details included age, sex, body and mass index. Clinical variables ascertained included hypertension, diabetes, presence of coronary artery disease, heart failure, chronic kidney disease, and Society for Thoracic Surgeons severity score. We recorded echocardiographic data including ejection fraction and aortic valve parameters. Lastly, procedure data included TAVR valve type, valve size, and whether procedure was valve-in-valve.

ECGs were performed prior to all TAVRs and immediately following the procedure, and on the day of discharge. Patients underwent 1-month follow-up either in the BIDMC TAVR clinic or with their local cardiologist, where a repeat ECG was done. As a result of the COVID19 pandemic, several patients underwent 1-month follow up via telehealth visits and therefore were unable to receive an ECG at that time.

We prospectively characterized several outcomes of interest prior to implementing the clinical protocol and initiating data collection. The primary clinical outcome was permanent pacemaker implantation prior to hospital discharge and at 1-month post-TAVR. Pre-specified secondary outcomes included delayed/late heart block, defined as heart block developing at least 2 days following TAVR or post-discharge. We also evaluated the ventricular pacing percentage for those patients that underwent pacemaker implantation; this was performed at their pacemaker clinic follow-up, which took place 1-4 weeks after implantation. Adverse events were noted including new conduction abnormalities and death prior to hospital discharge and at 1-month post-TAVR, including cause of death and whether this was correlated to conduction abnormality or procedural complication. Total length of stay and number of days spent in the critical care unit were also recorded.

In addition, we evaluated compliance with the clinical protocol and, for patients whose actual management appeared to differ, identified the specific ways in which actual versus recommended management diverged. 

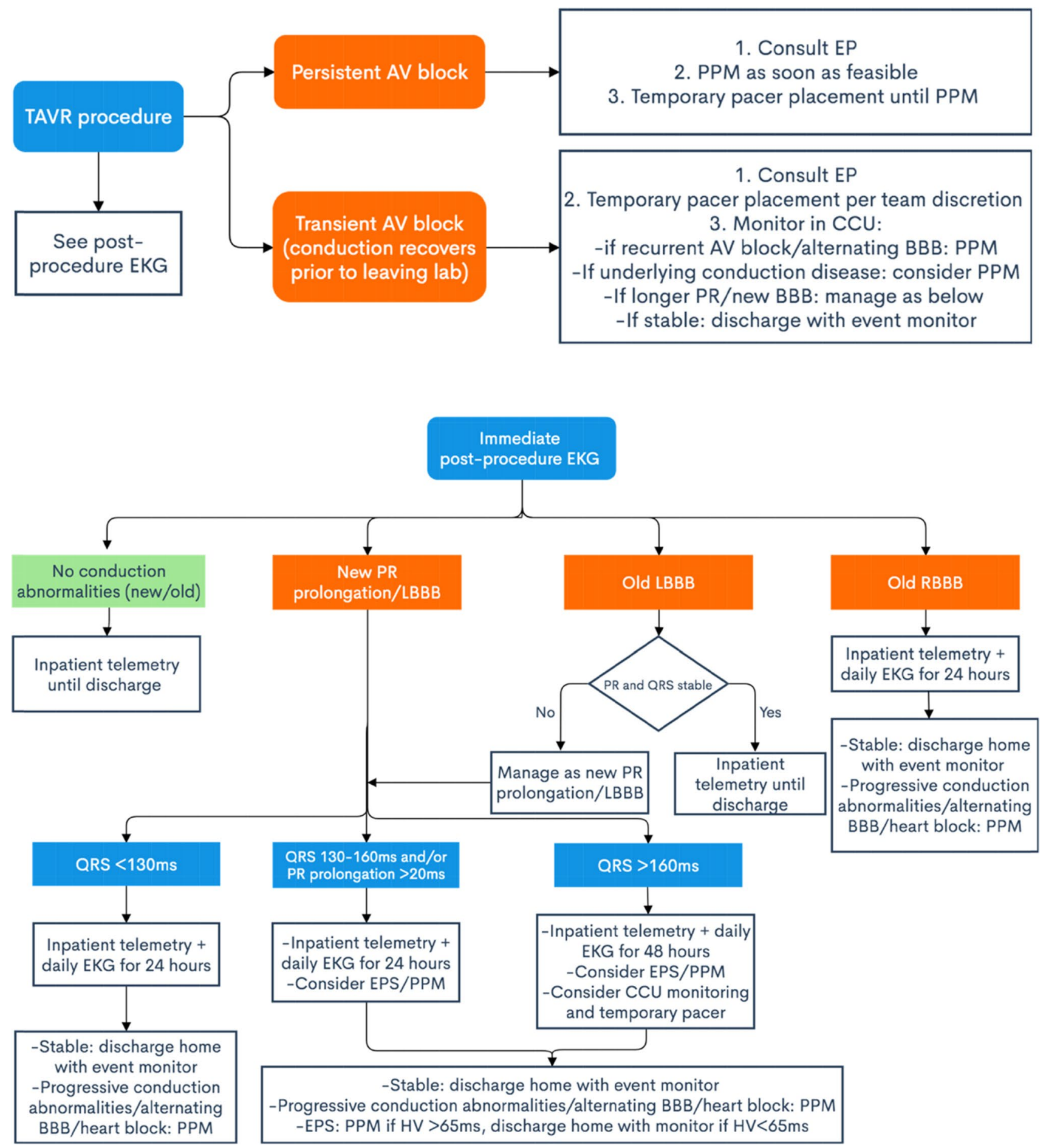

Fig. 1 Management pathway for patients undergoing TAVR

\subsection{Statistical analysis}

For variables describing the study population, categorical data were expressed as frequencies and percentages and continuous data as means and standard deviations. Categorical data were compared between the pacemaker and non-pacemaker groups using Fisher exact test, while twosided $t$-test was used to compare numerical data between these two groups. The proportion of patients receiving permanent pacemakers was identified, as well as their timing post-TAVR. Study adherence was evaluated by comparing 
the number of patients with protocol-concordant management to the overall cohort.

In an exploratory analysis, we evaluated the association between selected clinical factors (including pre-existing RBBB and AV delay, new LBBB, transient heart block, and valve type) and permanent pacemaker implantation at 1 month follow-up using unadjusted logistic regression to generate odds ratios $(O R)$ and $95 \%$ confidence intervals $(C I)$. Given the size of the study cohort, we decided a priori not to attempt multivariable regression to avoid model overfitting. All statistical analyses were performed using the Stata 13.0 software (College Station, TX).

\section{Results}

Of the 200 patients who underwent TAVR between February 2020 and February 2021, 19 were excluded due to pre-existing permanent pacemaker. Characteristics of the 181 patients used for analysis are summarized in Tables 1 and 2. Overall, the mean age was $77.9( \pm 9.1), 68(38 \%)$ were women, and common comorbidities included coronary artery disease
(52\%), hypertension (80\%), and hyperlipidemia (75\%). The most common type of valve utilized in our cohort was the balloon expandable Edwards SAPIEN 3 (73\%), followed by the Medtronic CoreValve Evolut Pro + $(25 \%)$, and the Boston Scientific Lotus Edge (2\%).

Dual-chamber pacemaker implantation for high grade or complete heart block occurred in 19 patients by hospital discharge, and 21 by 1 -month follow-up (13\%). Figure 2 shows the distribution of pacemaker implantation timing after TAVR. All pacemakers were implanted within 2 days of heart block development. For patients with persistent heart block during the TAVR, mean time to PPM was 0.7 days. Patients had a temporary pacemaker in place prior to PPM for an average of 1.1 days. Nine pacemakers were placed for persistent complete heart block that arose during the TAVR procedure and remained present at the procedure's completion. Of those, 5 underwent permanent pacemaker directly in the hybrid OR. The other 4 had temporary pacemakers placed with subsequent permanent pacemakers; further detailed chart review did not show a specific clinical reason for undergoing temporary pacemaker placement first, other than cardiac electrophysiology availability.
Table 1 Characteristics of Patients Undergoing TAVR overall and according to receipt of permanent pacemaker implantation (PPMI)

\begin{tabular}{|c|c|c|c|c|}
\hline & $\begin{array}{l}\text { All Patients } \\
(n=181)\end{array}$ & $\begin{array}{l}\text { PPMI } \\
(n=21)\end{array}$ & $\begin{array}{l}\text { No PPMI } \\
(n=160)\end{array}$ & $\begin{array}{l}p \text {-value } \\
\text { (PPPMI vs No } \\
\text { PPMI) }\end{array}$ \\
\hline Age, years & $77.9( \pm 9.1)$ & $78.5( \pm 9.0)$ & $77.8( \pm 9.1)$ & 0.740 \\
\hline Female & $68(38 \%)$ & $8(38 \%)$ & $60(38 \%)$ & 0.958 \\
\hline BMI, $\mathrm{kg} / \mathrm{m}^{2}$ & $28.5( \pm 6.2)$ & $28.2( \pm 4.6)$ & $28.5( \pm 6.4)$ & 0.819 \\
\hline $\begin{array}{l}\text { CAD } \\
\text { Prior MI } \\
\text { Prior PCI }\end{array}$ & $\begin{array}{l}94(52 \%) \\
25(14 \%) \\
42(23 \%)\end{array}$ & $\begin{array}{l}14(67 \%) \\
1(5 \%) \\
6(29 \%)\end{array}$ & $\begin{array}{l}80(50 \%) \\
24(15 \%) \\
36(23 \%)\end{array}$ & $\begin{array}{l}0.185 \\
0.074 \\
0.882\end{array}$ \\
\hline $\begin{array}{l}\text { Prior cardiac surgery } \\
\text { Prior CABG } \\
\text { Prior aortic valve surgery }\end{array}$ & $\begin{array}{l}33(18 \%) \\
23(13 \%) \\
14(8 \%)\end{array}$ & $\begin{array}{l}4(19 \%) \\
4(19 \%) \\
0(0 \%)\end{array}$ & $\begin{array}{l}29(18 \%) \\
19(12 \%) \\
14(9 \%)\end{array}$ & $\begin{array}{l}0.960 \\
0.353 \\
0.158\end{array}$ \\
\hline $\begin{array}{l}\text { Heart failure } \\
\text { Mean LVEF, \% }\end{array}$ & $\begin{array}{l}73(40 \%) \\
56( \pm 12)\end{array}$ & $\begin{array}{l}11(52 \%) \\
56( \pm 13)\end{array}$ & $\begin{array}{l}62(39 \%) \\
56( \pm 12)\end{array}$ & $\begin{array}{l}0.269 \\
0.744\end{array}$ \\
\hline Hypertension & $145(80 \%)$ & $17(81 \%)$ & $128(80 \%)$ & 0.918 \\
\hline Diabetes mellitus & $52(29 \%)$ & $10(48 \%)$ & $42(26 \%)$ & 0.042 \\
\hline Hyperlipidemia & $136(75 \%)$ & $20(95 \%)$ & $116(73 \%)$ & 0.023 \\
\hline Peripheral vascular disease & $21(12 \%)$ & $2(10 \%)$ & $19(12 \%)$ & 0.745 \\
\hline Atrial fibrillation & $50(28 \%)$ & $7(33 \%)$ & $43(27 \%)$ & 0.545 \\
\hline Stroke & $22(12 \%)$ & $5(24 \%)$ & $17(11 \%)$ & 0.082 \\
\hline COPD & $19(10 \%)$ & $2(10 \%)$ & $17(11 \%)$ & 0.877 \\
\hline $\begin{array}{l}\text { Chronic kidney disease } \\
\text { Dialysis }\end{array}$ & $\begin{array}{l}61(34 \%) \\
4(2 \%)\end{array}$ & $\begin{array}{l}6(29 \%) \\
1(5 \%)\end{array}$ & $\begin{array}{l}55(34 \%) \\
3(2 \%)\end{array}$ & $\begin{array}{l}0.584 \\
0.292\end{array}$ \\
\hline STS Score & $3.49( \pm 2.98)$ & $3.59( \pm 2.51)$ & $3.47( \pm 3.02)$ & 0.879 \\
\hline $\begin{array}{l}\text { Sapien3 } \\
\text { Evolut Pro + } \\
\text { Lotus Edge }\end{array}$ & $\begin{array}{l}132(73 \%) \\
45(25 \%) \\
4(2 \%)\end{array}$ & $\begin{array}{l}17(81 \%) \\
2(10 \%) \\
2(10 \%)\end{array}$ & $\begin{array}{l}115(72 \%) \\
43(27 \%) \\
2(1 \%)\end{array}$ & $\begin{array}{l}0.379 \\
0.084 \\
\mathbf{0 . 0 1 5}\end{array}$ \\
\hline $\begin{array}{l}\text { Mean AVA, } \mathrm{cm}^{2} \\
\text { Mean AV gradient, } \mathrm{mmHg} \\
\text { Peak AV gradient, } \mathrm{mmHg}\end{array}$ & $\begin{array}{l}0.78( \pm 0.27) \\
43.5( \pm 14.3) \\
70.5( \pm 23.2)\end{array}$ & $\begin{array}{l}0.77( \pm 0.19) \\
44.7( \pm 12.3) \\
75.5( \pm 21.6)\end{array}$ & $\begin{array}{l}0.79( \pm 0.27) \\
43.4( \pm 14.6) \\
69.8( \pm 23.4)\end{array}$ & $\begin{array}{l}0.808 \\
0.696 \\
0.297\end{array}$ \\
\hline
\end{tabular}

$P<0.05$ 
Table 2 Electrocardiographic features of study population

\begin{tabular}{|c|c|c|c|c|}
\hline & $\begin{array}{l}\text { All Patients } \\
(n=181)\end{array}$ & $\begin{array}{l}\text { PPMI } \\
(n=21)\end{array}$ & $\begin{array}{l}\text { No PPMI } \\
(n=160)\end{array}$ & $\begin{array}{l}p \text {-value } \\
\text { (PPMI vs No } \\
\text { PPMI) }\end{array}$ \\
\hline \multicolumn{5}{|c|}{ Baseline ECG features } \\
\hline 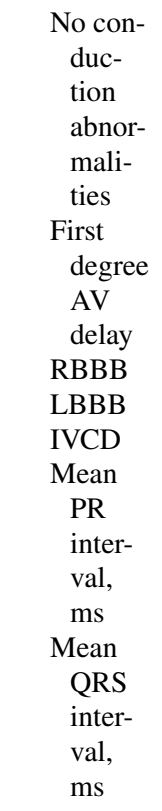 & $\begin{array}{l}109(60 \%) \\
41(23 \%) \\
30(17 \%) \\
17(9 \%) \\
14(8 \%) \\
187( \pm 38) \\
110( \pm 26)\end{array}$ & $\begin{array}{l}8(38 \%) \\
7(33 \%) \\
11(52 \%) \\
1(5 \%) \\
0(0 \%) \\
196( \pm 34) \\
124( \pm 30)\end{array}$ & $\begin{array}{l}101(63 \%) \\
34(21 \%) \\
19(12 \%) \\
16(10 \%) \\
14(9 \%) \\
186( \pm 38) \\
108( \pm 25)\end{array}$ & $\begin{array}{l}\mathbf{0 . 0 2 8} \\
0.119 \\
<\mathbf{0 . 0 0 1} \\
0.439 \\
0.158 \\
0.322 \\
\mathbf{0 . 0 0 8}\end{array}$ \\
\hline \multicolumn{5}{|c|}{ Discharge ECG features } \\
\hline $\begin{array}{l}\text { No con- } \\
\text { duc- } \\
\text { tion } \\
\text { abnor- } \\
\text { mali- } \\
\text { ties } \\
\text { First } \\
\text { degree } \\
\text { AV } \\
\text { delay } \\
\text { RBBB } \\
\text { LBBB } \\
\text { IVCD } \\
\text { Mean } \\
\text { PR } \\
\text { inter- } \\
\text { val, } \\
\text { ms } \\
\text { Mean } \\
\text { QRS } \\
\text { inter- } \\
\text { val, } \\
\text { ms }\end{array}$ & $\begin{array}{l}66(36 \%) \\
41(23 \%) \\
20(11 \%) \\
34(19 \%) \\
14(8 \%) \\
190( \pm 38) \\
119( \pm 29)\end{array}$ & $\begin{array}{l}1(5 \%) \\
9(43 \%) \\
4(19 \%) \\
3(14 \%) \\
0(0 \%) \\
204( \pm 20) \\
149( \pm 24)\end{array}$ & $\begin{array}{l}65(41 \%) \\
32(20 \%) \\
16(10 \%) \\
31(19 \%) \\
14(9 \%) \\
188( \pm 39) \\
115( \pm 27)\end{array}$ & $\begin{array}{l}\mathbf{0 . 0 0 1} \\
\mathbf{0 . 0 1 9} \\
0.214 \\
0.575 \\
0.158 \\
0.124 \\
<\mathbf{0 . 0 0 1}\end{array}$ \\
\hline
\end{tabular}

$R B B B$, right bundle branch block; $L B B B$, left bundle branch block; $I V C D$, intraventricular conduction block

$P<0.05$

Twelve total patients had transient peri-procedural complete heart block. Of those, one underwent direct permanent pacemaker placement in the hybrid OR (noted to have complete heart block for 15 min during TAVR) and another (with pre-existing RBBB and LAFB) underwent pacemaker placement later that day. Three had temporary wires placed; of those 2 had pre-existing AV delay and RBBB and, despite AV recovery after TAVR, underwent pacemaker placement as they were deemed high risk. One was noted to have low valve implantation depth in relation to the membranous septum and developed high grade AV block following TAVR, resulting in permanent pacemaker placement. No other patients with transient heart block during the procedure developed delayed heart block.

Four patients received pacemakers for advanced conduction disease arising after the procedure was completed, but before 2 days had passed. One patient with no pre-procedure conduction disease developed intermittently regularized atrial fibrillation with ventricular rates in the $30 \mathrm{~s}$ (narrow QRS) the same day as the TAVR and had a pacemaker placed that day. A second patient with atrial fibrillation and LBBB at baseline developed regularized complete heart block with a LBBB escape post-procedure (same day) and received a pacemaker. A third patient with a pre-existing RBBB had 2:1 and then complete heart block immediately after the TAVR procedure but technically after the procedure was completed; this patient received a permanent pacemaker the same day. The fourth patient in this group had syncope with 20 s of complete heart block and no ventricular escape the day after their procedure, and had a pacemaker placed.

Three patients $(2 \%)$ developed delayed complete heart block ( $>2$ days after TAVR) and all had received Sapien valves. Two of those 3 patients developed heart block after hospital discharge, and both had been discharged with a cardiac monitor due to pre-existing RBBB. The first patient developed pre-syncopal symptoms and heart block 17 days after TAVR, after his 2-week cardiac monitoring period, which had shown no heart block or other conduction abnormalities. The second patient was found to have heart block on cardiac monitoring 4 days after TAVR and was asymptomatic.

Two other patients in the cohort had pacemaker implantation within 1 month of hospital discharge, but one was for sinus bradycardia and the other for CRT-D placement.

Thirty patients had a pre-existing RBBB, 40 developed a new LBBB (4\% were transient LBBB not present on ECG immediately after TAVR) and 23 developed PR prolongation $>20 \mathrm{~ms}$. Within these groups, permanent pacemaker implantation rate was $37 \%, 13 \%$, and $17 \%$, respectively. Only one patient in the cohort underwent EPS for a wide QRS post-TAVR $>160 \mathrm{~ms}$ and transient heart block. He was found to have an $\mathrm{HV}$ interval of $75 \mathrm{~ms}$ and underwent pacemaker placement as a result.

Ventricular pacing data was available for 18 of the 21 patients that underwent pacemaker implantation. All patients were programmed with manufacturer-specific algorithms intended to promote intrinsic conduction. 
Fig. 2 Time from TAVR to PPM implantation by valve type

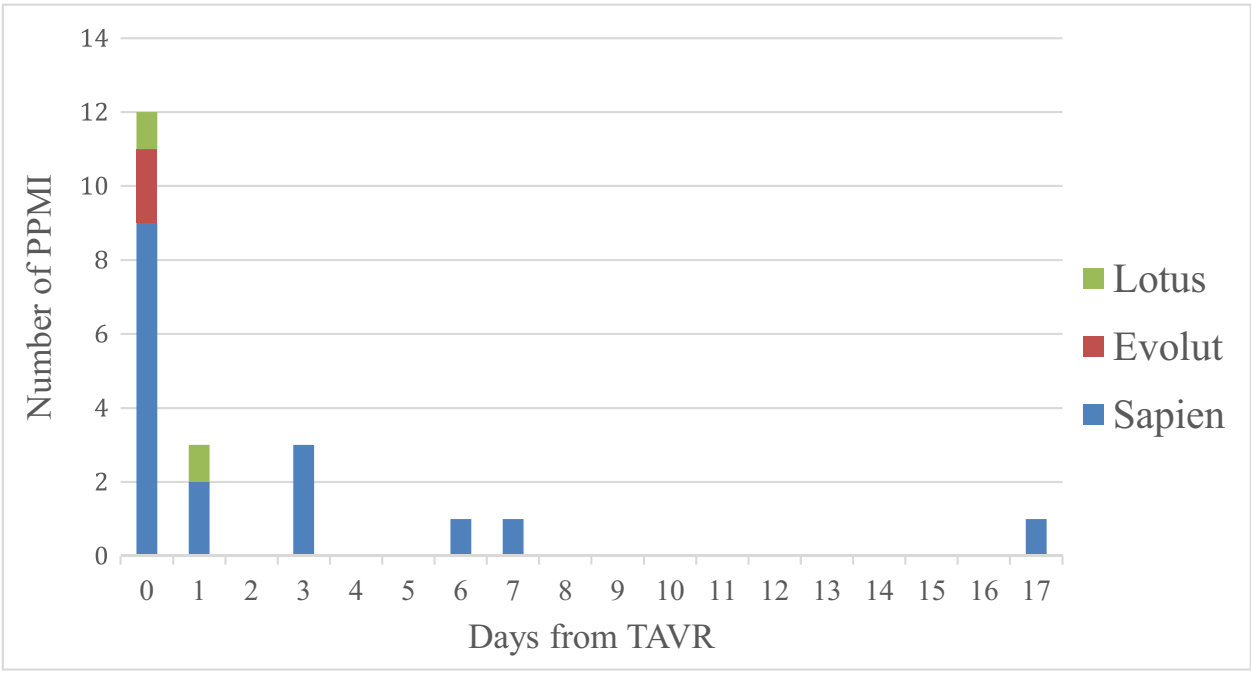

Interrogation took place at 1-4 weeks and revealed that only 1 patient had no ventricular pacing, 2 patients had $<1 \%$ ventricular pacing, and mean ventricular pacing for the other 15 patients was $72 \%$. In our exploratory analysis, pre-existing RBBB, transient peri-procedural heart block, and LOTUS valves were associated with pacemaker implantation albeit with wide $C I$ reflective of the modest sample size, with $O R(C I)$ of 8.16 (3.06-21.78), 6.83 (1.94-24.03), and 8.32 (1.11-62.49), respectively. Preexisting AV delay, new LBBB, and the Evolut valve were not associated with new pacemakers (Table 3 ).

There were 7 deaths (4\%) by 1 month follow-up. Two patients died of post-procedural vascular complications. There were no pacemaker implantation related complications. None of the deaths were linked to syncope or conduction abnormality. Average length of stay was 3.0 days $( \pm 2.7)$. Twenty-three patients required hospitalization in the critical care unit, and length of stay for those patients was 3.0 days $( \pm 2.1)$.

Overall, $93 \%$ of the 181 patients were managed according to our clinical pathway; those that were not were due to failure of discharge with a heart monitor when it was

Table 3 Factors associated with permanent pacemaker implantation

\begin{tabular}{lll}
\hline & Unadjusted $\boldsymbol{O R}(95 \% \boldsymbol{C I})$ & $p$-value \\
\hline Pre-existing RBBB & $8.16(3.06-21.78)$ & $<\mathbf{0 . 0 0 1}$ \\
Transient heart block & $6.83(1.94-24.03)$ & $\mathbf{0 . 0 0 3}$ \\
Pre-existing AV delay & $2.29(0.79-6.61)$ & 0.127 \\
Sapien3 & $1.66(0.53-5.21)$ & 0.38 \\
Evolut Pro+ & $0.29(0.64-1.28)$ & 0.102 \\
Lotus Edge & $8.32(1.11-62.49)$ & $\mathbf{0 . 0 4 0}$ \\
New LBBB & $0.84(0.27-2.66)$ & 0.767 \\
\hline
\end{tabular}

$P<0.05$ clinically indicated for either pre-existing $\mathrm{RBBB}$ or new PR prolongation/new LBBB.

\section{Discussion}

This single-center prospective evaluation of a standardized protocol for the management of conduction abnormalities after TAVR includes several key observations. First, a multispecialty protocol can be implemented with high compliance, achieving standardization of care for a complex decision with important trade-offs. Second, this pathway yielded moderate pacemaker implantation rates, while illustrating the safety of early hospital discharge with a strategy of targeted monitoring of high-risk patients. Lastly, our findings support prior observations that delayed heart block after TAVR is uncommon and typically identified by cardiac monitoring among asymptomatic patients.

This study builds on prior reports seeking consensus to address the management of conduction abnormalities after TAVR [6-8]. Our pathway, compared to those outlined by Rodés-Cabau, Josep et al., and Auffret, Vincent et al., makes use of 14-day outpatient cardiac monitoring and favors telemetry monitoring over maintaining transvenous pacing for patients with new PR or QRS prolongation with preexisting conduction disease. Our approach may have facilitated shorter hospital stays (with an average LOS similar to that reported in the 2019 TVT registry [2]) and fewer or no days spent in the critical care unit for temporary pacemaker monitoring, both of which were explicit targets in our pathway's initial formulation. Notably, ACC consensus guidelines released halfway through our study highlight a similar risk stratification of patients to that of our pathway, but favor a more conservative management for those patients with new or worsened conduction disturbance after TAVR (PR or QRS interval increase $\geq 10 \%$ ), with concern that early 
discharge after TAVR is less likely to be safe [6]. Reassuringly, we observed no morbidity or mortality correlated to conduction abnormalities in the outpatient setting despite early discharges.

The incidence and risk factors of delayed heart block are still not well understood and the optimal way to monitor these patients in the era of early hospital discharge remains an important clinical question. We report a rate of delayed heart block of $2 \%$, which is in fact lower than previously reported rates ranging from 4.6 to $8 \%$ [9-11]. There may be several reasons for the discrepancy in observed rates, including variable definitions of delayed heart block by study, differences in valve type and structural factors like implantation depth, and, in our study, failure to detect asymptotic episodes of heart block in low-risk patients who did not receive an ECG at 1-month follow-up and were not discharged with a cardiac monitor. Nevertheless, our study shows that delayed heart block remains a rare complication, and that none of the low-risk patients who were discharged without a cardiac monitor had any morbidity or mortality related to heart block.

Though modestly sized, our study highlighted several potential risk factors for pacemaker implantation that may merit continued prospective investigation. In our sample, patients with pre-existing RBBB had 8 times higher odds of pacemaker implantation than those without. Both patients who developed heart block after their hospitalization also had pre-existing RBBB, which has previously been identified as a predictor for delayed heart block specifically [10, 12]. Despite known risk factors such as Evolut valves and new LBBB $[8,10,13,14]$ being common in our cohort, we did not find an association between either and pacemaker implantation. Lotus valve use was rare in our cohort, making conclusions unreliable, but we found that these patients had eightfold higher odds of pacemaker implantation. Reports on Lotus valves are more sparse compared to the Sapien and Corevalve systems, but two prior studies have in fact demonstrated high pacemaker rates in this population as well, approximating pacemaker rates of $30 \%[15,16]$. While the Lotus Edge valve has since been recalled, we encourage further investigation into the next generation of valves that utilize similar delivery systems.

Our study includes specific limitations. First, the study cohort, being at a large academic teaching hospital, is subject to referral bias and the results may not be generalizable to other clinical settings. For example, our pathway favors telemetry monitoring over maintaining transvenous pacing in certain patients but doing so relies on a center's ability to establish emergency temporary pacing if needed. Second, due to the COVID-19 pandemic, complete 1-month followup data was not available as several of our patients underwent telehealth follow-up visits and therefore were unable to have ECGs performed. This, along with the possibility of subclinical conduction disease occurring after the monitoring period, could result in under-detection of delayed heart block in low-risk asymptomatic patients. However, the risk of delayed heart block in a patient with a baseline normal ECG and no new conduction abnormalities has been reported to be $<1 \%$; therefore, it is unlikely that cases in our sample were missed. Third, our study was not powered to detect correlations between risk factors and pacemaker implantation, and, due to our overall small sample size, conclusions about risk factors should be interpreted as observational findings. Relatedly, due to evolving changes in TAVR technology, operator experience, and patient selection factors, we a priori chose not to attempt comparisons across institutions or within our own institutional practice (e.g., in a pre/post format) due to the difficult in drawing causal inference regarding the impact of our pathway. Nevertheless, we hope these findings illustrate the feasibility of a standardized protocol and provide a benchmark for future work and expectations. For patients receiving pacemakers, our description of pacing percentage at follow-up necessarily provides only a snapshot of pacing-dependence given subtle differences in programming, and the possibility that certain conduction patterns such as paroxysmal AV block may yield low pacing burdens despite representing true pacemakerdependence in clinical terms.

In summary, this report illustrates that a standardized protocol for the management of conduction abnormalities after TAVR can be implemented with high compliance, safe management of conduction disturbance, and relatively brisk discharge supported by ambulatory monitoring.

\section{Declarations}

Conflict of interest The authors declare no competing interest.

\section{References}

1. Khatri PJ, Webb JG, Rodes-Cabau J, et al. Adverse effects associated with transcatheter aortic valve implantation: a meta-analysis of contemporary studies. Ann Intern Med. 2013;158(1):35-46.

2. Carroll JD, Mack MJ, Vemulapalli S, et al. STS-ACC TVT Registry of transcatheter aortic valve replacement. J Am Coll Cardiol. 2020;76(21):2492-516.

3. Mack MJ, Leon MB, Thourani VH, et al. Transcatheter aorticvalve replacement with a balloon-expandable valve in low-risk patients. N Engl J Med. 2019;380(18):1695-705.

4. Popma JJ, Deeb GM, Yakubov SJ, et al. Transcatheter aortic-valve replacement with a self-expanding valve in low-risk patients. $\mathrm{N}$ Engl J Med. 2019;380(18):1706-15.

5. Kawsara A, Sulaiman S, Alqahtani F, et al. Temporal trends in the incidence and outcomes of pacemaker implantation after transcatheter aortic valve replacement in the United States (20122017). J Am Heart Assoc. 2020;9(18):e016685. 
6. Lilly SM, Deshmukh AJ, Epstein AE, et al. 2020 ACC expert consensus decision pathway on management of conduction disturbances in patients undergoing transcatheter aortic valve replacement: a report of the American College of Cardiology Solution Set Oversight Committee. J Am Coll Cardiol. 2020;76(20):2391-411.

7. Rodes-Cabau J, Ellenbogen KA, Krahn AD, et al. Management of conduction disturbances associated with transcatheter aortic valve replacement: JACC scientific expert panel. J Am Coll Cardiol. 2019;74(8):1086-106.

8. Auffret V, Puri R, Urena M, et al. Conduction disturbances after transcatheter aortic valve replacement: current status and future perspectives. Circulation. 2017;136(11):1049-69.

9. Toggweiler S, Stortecky S, Holy E, et al. The electrocardiogram after transcatheter aortic valve replacement determines the risk for post-procedural high-degree AV block and the need for telemetry monitoring. JACC Cardiovasc Interv. 2016;9(12):1269-76.

10. Ream K, Sandhu A, Valle J, et al. Ambulatory rhythm monitoring to detect late high-grade atrioventricular block following transcatheter aortic valve replacement. J Am Coll Cardiol. 2019;73(20):2538-47.

11. Tian Y, Padmanabhan D, McLeod CJ, et al. Utility of 30-day continuous ambulatory monitoring to identify patients with delayed occurrence of atrioventricular block after transcatheter aortic valve replacement. Circ Cardiovasc Interv. 2019;12(12):e007635.
12. Mangieri A, Lanzillo G, Bertoldi L, et al. Predictors of advanced conduction disturbances requiring a late $(>/=48 \mathrm{~h})$ permanent pacemaker following transcatheter aortic valve replacement. JACC Cardiovasc Interv. 2018;11(15):1519-26.

13. Schymik G, Tzamalis P, Bramlage $P$, et al. Clinical impact of a new left bundle branch block following TAVI implantation: 1-year results of the TAVIK cohort. Clin Res Cardiol. 2015;104(4):351-62.

14. Urena M, Webb JG, Cheema A, et al. Impact of new-onset persistent left bundle branch block on late clinical outcomes in patients undergoing transcatheter aortic valve implantation with a balloonexpandable valve. JACC Cardiovasc Interv. 2014;7(2):128-36.

15. Zaman S, McCormick L, Gooley R, et al. Incidence and predictors of permanent pacemaker implantation following treatment with the repositionable Lotus transcatheter aortic valve. Catheter Cardiovasc Interv. 2017;90(1):147-54.

16. Falk V, Wohrle J, Hildick-Smith D, et al. Safety and efficacy of a repositionable and fully retrievable aortic valve used in routine clinical practice: the RESPOND Study. Eur Heart J. 2017;38(45):3359-66.

Publisher's note Springer Nature remains neutral with regard to jurisdictional claims in published maps and institutional affiliations. 